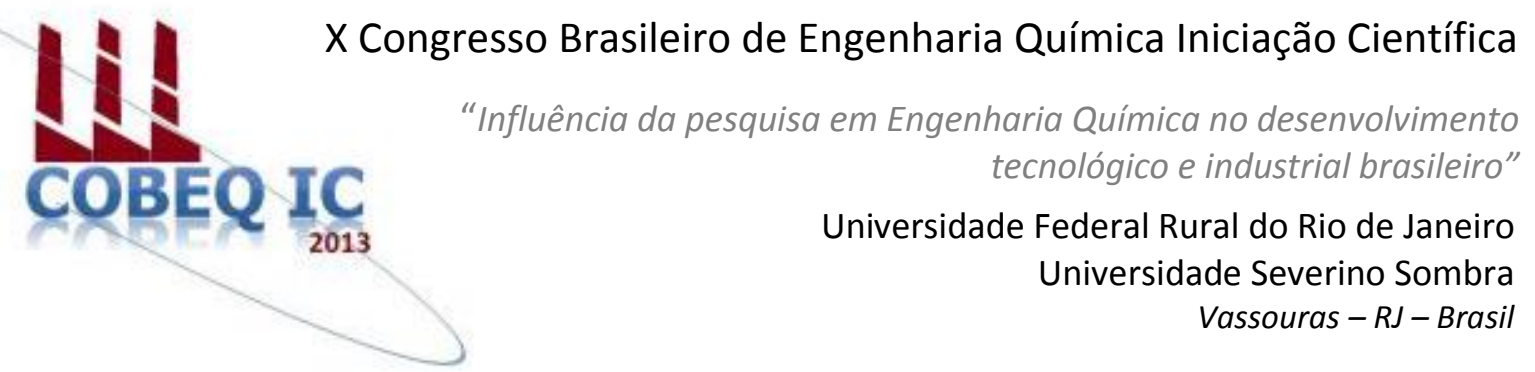

\title{
DESENVOLVIMENTO DE NOVOS PRODUTOS DA MACADÂMIA
}

\author{
SILVA* $^{1}$, T. C. A.; ARRIECHE ${ }^{2}$, L. S.; SARTORI ${ }^{3}$ D. J. M. \\ ${ }^{1}$ Thamires Canuto de Almeida e Silva do DETEC/UFES $\quad{ }^{2}$ Professor do DETEC/UFES \\ Departamento de Engenharias e Tecnologia - CEUNES - Universidade Federal do Espírito Santo \\ ${ }^{3}$ Universidade Federal de São Carlos, Departamento de Engenharia Química \\ Endereço-Rodovia BR 101 Norte, Km. 60, Bairro Litorâneo, São Mateus, CEP. 29932-540, ES, \\ email: leonardoarrieche@ceunes.ufes.br
}

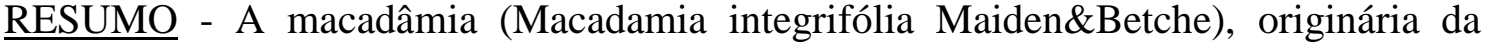
Austrália, apresenta frutos lisos e arredondados, esta nogueira produz nozes de excelente sabor, as quais são utilizadas "in natura" acompanhando aperitivos, na confecção de doces, sendo bem aceitas pela população. Neste trabalho, foi analisado o processo de produção da macadâmia na Cooperativa Agroindustrial dos Produtores de Noz Macadâmia (Coopmac), localizada em São Mateus, com a finalidade de identificar oportunidades de desenvolvimento de novos produtos. Foram exploradas etapas de: análise do procedimento atual; geração de ideias; desenvolvimento de conceito de produto. Como o óleo de macadâmia era apenas extraído e não havia qualquer forma de processamento ou análise, decidiu-se a partir deste desenvolver um produto potencial. Inicialmente foram realizadas análises químicas deste óleo e optou-se pela produção de sabonetes líquidos e em barra. Durante esta etapa, a metodologia de planejamento de experimentos foi utilizada no desenvolvimento do protótipo e a análise sensorial, a partir da escala hedônica do tipo LAM, foi utilizada nos testes. Feito os testes, os dados foram analisados e pode-se distinguir a melhor formulação e os efeitos principais em cada quesito avaliado. Espera-se contribuir com a geração de conhecimento científico e tecnológico à produção de macadâmia no Espírito Santo.
\end{abstract}

Palavras chave: planejamento fatorial, análise sensorial, óleo.

\section{INTRODUÇÃO}

A macadâmia (Macadamia integrifólia Maiden\&Betche), originária da Austrália e pertencente à família Proteaceae, apresenta frutos lisos, esféricos ou arredondados. No Brasil, esta espécie foi introduzida somente em 1931, primeiramente na região de Limeira, sendo atualmente encontrada em várias regiões do Estado de São Paulo (Dierberger \& Marino Netto, 1985).

A cultura da macadâmia destaca-se como atividade promissora com vistas ao mercado externo. O Brasil está em $6^{\circ}$ lugar na produção mundial, com cerca de $3 \%$ da produção. Há uma demanda crescente pelo produto, o que vem atraindo a atenção de investidores neste ramo do agronegócio. 
A amêndoa é rica em óleos mono insaturados, que são disputados pela indústria de cosméticos na composição de hidratantes e por laboratórios farmacêuticos como redutor dos níveis de colesterol. Os ácidos graxos normalmente relatados em nozes macadâmia são: mirístico (0,6-1,8\%), palmítico (7-24\%), palmitoléico (15-30\%), esteárico (2-5\%), oléico (40-65\%), linoléico (1,4-4,5\%) e araquídico (1,2-4,5\%).

$\mathrm{O}$ beneficiamento atual da macadâmia na Cooperativa Coopmac consiste em apenas a produção do produto simples e embalado (noz integral). No entanto, é possível identificar novas oportunidades e formas de processamento a partir da macadâmia, a fim de gerar maior lucro aos cooperados.

$\mathrm{O}$ processo de desenvolvimento de novos produtos, destinado à descoberta e incremento de produtos, é constituído de oito passos principais. Esses passos são mostrados na Figura 1 (Kotler,1993).

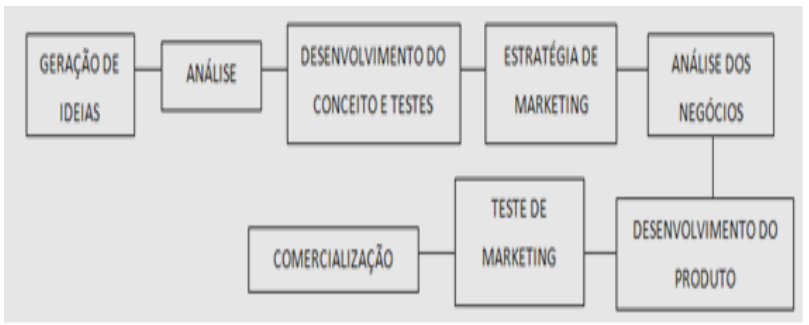

Figura 1 - Principais etapas no

desenvolvimento de um novo produto.

A análise sensorial é uma ferramentachave no desenvolvimento de produtos. Os testes necessários devem ser aplicados conforme os critérios do produto que se deseja avaliar. Um bom planejamento dos testes, uma criteriosa seleção dos julgadores e uma correta interpretação dos testes são fatores muito importantes para obter respostas confiáveis para a finalidade de desenvolver um produto novo de qualidade e de aceitação favorável.

\section{OBJETIVOS}

Neste trabalho será analisado o processo de produção e beneficiamento da macadâmia, com a finalidade de identificar novas oportunidades de desenvolvimento de novos produtos a partir da macadâmia. Serão exploradas as etapas de: análise do procedimento atual; geração de ideias; desenvolvimento de conceito de produto, parâmetros físicos e nutricionais e o desenvolvimento de um protótipo do produto. Durante estas etapas, a análise sensorial será utilizada como uma das ferramentas básicas na determinação do melhor protótipo.

\section{METODOLOGIA}

Este trabalho avaliará a macadâmia produzida pela Cooperativa Agroindustrial dos Produtores de Noz Macadâmia (Coopmac), que fica localizada na cidade de São Mateus, no norte do Espírito Santo, responsável por beneficiar e comercializar a produção dos 23 associados do Espírito Santo e da Bahia, com relação às oportunidades de desenvolvimento de novos produtos, com base em análise sensorial para o desenvolvimento do protótipo. Mais de $90 \%$ da produção local é destinada ao mercado internacional, sendo vendida principalmente para os Estados Unidos, o maior comprador da produção local (Agrosoft, 2010).

O desenvolvimento do protótipo seguirá as seguintes etapas:

- Análise do processo atual

- Etapas do desenvolvimento de novos produtos: geração de ideias; análise de ideias; desenvolvimento do conceito de produto e desenvolvimento do protótipo.

A metodologia utilizada neste protótipo é a de planejamento de experimentos, esta permite ao experimentador melhorar as performances dos produtos e processos, atenuar a sensibilidade de seus elementos a fatores que não podem ser controlados e reduzir os custos de desenvolvimento. Seu princípio básico permite variar de uma só vez todos os níveis de todas as variáveis, chamadas de fatores, a cada experiência, de maneira programada e racional. Entre todos os fatores estudados, apenas alguns apresentam influência significativa na variação das respostas.

Experimentos fatoriais são aqueles que incluem todas as combinações de vários conjuntos de tratamentos ou fatores. É indicado quando se deseja estudar os efeitos de duas ou mais variáveis de influência. Normalmente codificam-se os níveis dos fatores com os sinais (+) atribuídos ao nível superior e (-) atribuído ao nível inferior. Um 
experimento fatorial completo ocorre quando todas as possíveis combinações de níveis dos fatores são testadas. (Werkema 1996).

Feito o planejamento, análises sensoriais foram efetuadas com os resultados obtidos.

A qualidade do sabão foi analisada por meio de uma escala hedônica, que consiste basicamente em apresentar as amostras dos produtos, de maneia inteiramente ao acaso, aos provadores e pergunta-lhes sobre a preferência entre elas, segundo uma escala estabelecida, baseada nos atributos gosta e desgosta.

Os pontos da escala são distinguidos verbalmente, de modo que possam ser associados a valores numéricos, possibilitando análise estatística dos resultados.

O tipo de escala utilizada foi a escala LAM (Labeled Affective Magnitude), mostrada na figura 2, onde os espaços determinam a magnitude de aceitação, onde até mesmo os avaliadores, depois de avaliarem, podem ter uma ideia da diferença de uma amostra e outra ao visualizarem o esquema das escalas.

Os provadores foram instruídos sobre as principais características de qualidade do produto em estudo. Os quesitos avaliados foram: aparência; odor; limpeza; espuma e ação hidratante.

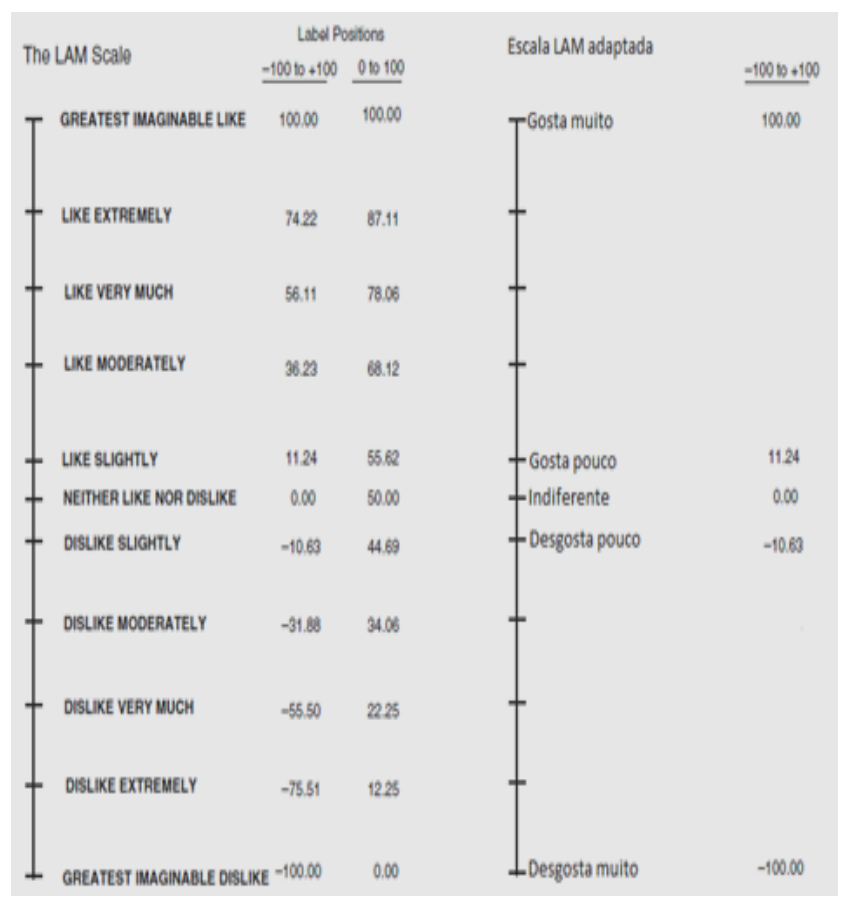

Figura 2 - Escala LAM e escala LAM adaptada utilizada nos testes de aceitação.
A análise descritiva quantitativa (ADQ) foi o método de análise utilizado no protótipo. Tal método avalia a intensidade dos atributos sensoriais presentes no produto através de uma escala hedônica, esta mede o gostar ou desgostar de um produto. A avaliação da escala hedônica foi convertida em escores numéricos que foram analisados estatisticamente para determinar a diferença no grau de preferência entre amostras (IFT, 1981; Land e Shepherd, 1988; ABNT, 1998).

Análises químicas, em laboratório, também foram utilizadas no desenvolvimento do protótipo.

\section{RESULTADOS}

1-Análise do processo atual

Fluxograma do processo na Cooperativa

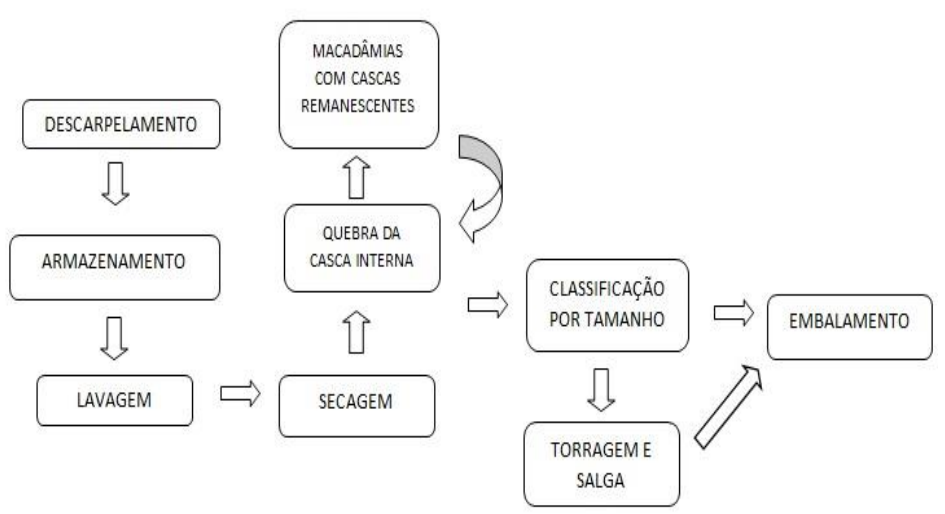

Figura 3 - Fluxograma do processo atual da Coopmac. Fonte: Thamires Canuto

2- Geração de ideias e desenvolvimento de conceito de produto

- Geração de ideias:

Diante de um público alvo, dos benefícios que o produto pode vir a oferecer, da necessidade de aprimoramento de técnicas já utilizadas, desenvolvimento de novos produtos, foram geradas ideias, a partir das já existentes e até mesmo novas ideias de desenvolvimento de produtos de macadâmia que possam ser adaptadas ao processo atual da Cooperativa.

- Análise de ideias:

A análise tem como objetivo separar as ideias fracas das ideias promissoras, aquelas que gerem lucros e boa produtividade. É necessária a escolha das ideias que possam ser implantadas no processo de produção da 
macadâmia de uma forma que os custos de inclusão sejam compensados pelos lucros de mercado devido à venda do produto desenvolvido.

$\mathrm{Na}$ Cooperativa o produto in natura é o alvo da produção, o único processamento é a torra e salga da noz, no entanto em menor escala de comercialização. Como o óleo, figura 4, é apenas extraído e não há qualquer forma de processamento ou análise, decidiu-se então fazer a análise do óleo extraído da macadâmia e a partir desta desenvolver um produto potencial.

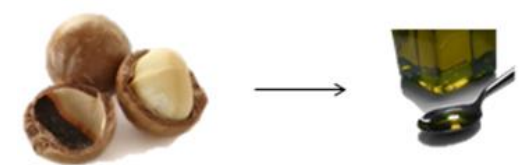

Figura 4 - Ideia escolhida.

- Desenvolvimento do conceito de produto e testes

O produto desenvolvido tem como base o óleo extraído da macadâmia, a partir de análises químicas identificou-se a composição deste. A principal ideia se resumiu ao desenvolvimento de cosméticos a partir das características encontradas neste óleo.

\section{Análises químicas}

Determinação do índice de acidez

O índice de acidez é definido como o numero de $\mathrm{mg}$ de hidróxido de potássio/sódio necessário para neutralizar um grama da amostra e revela o estado de conservação do óleo. O IA apresentou o valor de 10,893 $\mathrm{mgNaOH} / \mathrm{g}$ e o $\mathrm{AG}$ foi de $5,474 \%$, onde $\mathrm{AG}$ é a porcentagem de ácidos graxos livres, expressa como ácido oleico, em óleos comuns, brutos e refinados.

Determinação do índice de peróxido

Este método determina todas as substâncias, em termos de miliequivalentes de peróxido por $1000 \mathrm{~g}$ de amostra, que oxidam o iodeto de potássio nas condições do teste. No entanto, no procedimento utilizado a quantidade de peróxidos presentes no óleo foi muito baixa e não pode ser identificada.

Determinação de índice de saponificação

$O$ índice de saponificação é a quantidade de álcali necessário para saponificar uma quantidade definida de amostra. O IS obtido foi de 173,577
$\mathrm{mgKOH} / \mathrm{g}$. O Índice de Saponificação também indica o peso molecular médio dos ácidos graxos presentes em um óleo ou gordura, o PM médio dos ácidos graxos presentes obtido foi de 967,870 .

Planejamento Fatorial Sabonete em barra

Formulação analisada

$100 \mathrm{~g}$ de base glicerinada

$9 \mathrm{~mL}$ de mel

1,5/3,0 mL de óleo de macadâmia

$1,5 / 3,0 \mathrm{~g}$ de argila branca

$1 / 3 \mathrm{~mL}$ de lauril/cocoamido (50\%)

$0,5 \mathrm{~g}$ de conservante

$0,1 \mathrm{~g}$ de corante

$5 \mathrm{~mL}$ de essência

Planejamento $2^{3}$

A variável de resposta a ser determinada neste projeto foi a qualidade do sabonete produzido a partir do óleo de macadâmia, os fatores são o volume de óleo, volume de lauril e massa de argila, em dois níveis diferentes cada.

Tabela 1 - Valores dos níveis no planejamento final.

\begin{tabular}{ccc}
\hline $\begin{array}{c}\text { Volume de } \\
\text { óleo(mL) }\end{array}$ & $\begin{array}{c}\text { Volume 50\% de } \\
\text { lauril/cocoamido(mL) }\end{array}$ & $\begin{array}{c}\text { Massa de } \\
\text { argila } \\
\text { branca(g) }\end{array}$ \\
\hline $\mathrm{X}_{1}=1,5$ & $\mathrm{Y}_{1}=0,5 / 0,5$ & $\mathrm{Z}_{1}=1,5$ \\
$\mathrm{X}_{2}=3,0$ & $\mathrm{Y}_{2}=1,5 / 1,5$ & $\mathrm{Z}_{2}=3,0$ \\
\hline
\end{tabular}

Trabalhando com variáveis codificadas temos:

\section{Tabela 2 - Planejamento final $2^{3}$ sabonete em barra.}

\begin{tabular}{lccc}
\hline Experimento & Volume & Volume de & Massa \\
& de óleo & lauril/cocoamido & de \\
& $(\mathrm{mL})$ & $(\mathrm{mL})$ & argila \\
& & & branca
\end{tabular}

(g)

\begin{tabular}{llll}
\hline $\mathbf{1}$ & -1 & -1 & -1 \\
$\mathbf{2}$ & +1 & -1 & -1 \\
$\mathbf{3}$ & +1 & +1 & -1 \\
$\mathbf{4}$ & -1 & +1 & +1 \\
$\mathbf{5}$ & +1 & +1 & +1 \\
$\mathbf{6}$ & -1 & +1 & -1 \\
$\mathbf{7}$ & -1 & -1 & +1 \\
$\mathbf{8}$ & +1 & -1 & +1 \\
\hline
\end{tabular}


Planejamento Fatorial Sabonete Líquido

Formulação analisada

$250 \mathrm{~mL}$ de base para sabonete líquido

$750 \mathrm{~mL}$ de água destilada

7,5 mL de essência

4 gotas de corante

$10 \mathrm{~g}$ de cloreto de sódio

$5 / 10 \mathrm{~mL}$ de anfótero de coco

5/10 mL de óleo de macadâmia

Planejamento $2^{2}$

Tabela 3 - Níveis do planejamento $2^{2}$ sabonete líquido.

\begin{tabular}{ccrc}
\hline \multicolumn{2}{c}{ Volume de óleo $(\mathbf{m L})$} & \multicolumn{3}{c}{ Volume anfótero $(\mathbf{m L})$} \\
\hline $\mathrm{X}_{1}=5$ & $\mathrm{X}_{2}=10$ & $\mathrm{Y}_{1}=5$ & $\mathrm{Y}_{2}=10$ \\
\hline
\end{tabular}

Trabalhando com variáveis codificadas temos:

Tabela 4 - Planejamento fatorial 22 sabonete líquido.

\begin{tabular}{ccc}
\hline Experimento & $\begin{array}{c}\text { Volume de } \\
\text { óleo }(\mathbf{m L})\end{array}$ & $\begin{array}{c}\text { Volume de } \\
\text { anfótero }(\mathbf{m L})\end{array}$ \\
\hline $\mathbf{1}$ & +1 & +1 \\
$\mathbf{2}$ & -1 & +1 \\
$\mathbf{3}$ & +1 & -1 \\
$\mathbf{4}$ & -1 & -1 \\
\hline
\end{tabular}

\section{3 - Discussão}

Os testes foram realizados com 30 voluntários com as amostras aleatoriamente apresentadas e sem a identificação da formulação, os dados obtidos a partir destes foram analisados com o software Statistica e com base em cálculos de médias a partir dos valores estabelecidos pela escala.

A partir das médias, considerando os valores de $-100 \mathrm{a}+100$, foi criada uma variável complementar com a média geral destas médias ponderadas. A variável complementar foi definida como qualidade global, apresentada nas Tabelas 5 e 6, e a partir dela foi possível identificar a composição de maior aceitação.

Tabela 5 - Médias Sabonetes Líquidos

\begin{tabular}{lcccc}
\hline AMOSTRA & $\mathbf{1}$ & $\mathbf{2}$ & $\mathbf{3}$ & $\mathbf{4}$ \\
\hline APARÊNCIA & 5,68 & 66,81 & 17,85 & 37,49 \\
ODOR & 4,93 & 9,33 & 31,12 & 26,74 \\
\hline
\end{tabular}

\begin{tabular}{lcccc}
\hline ESPUMA & $-5,4$ & 66,7 & 70,41 & 27,83 \\
LIMPEZA & 53,37 & 59,75 & 78,91 & 90,75 \\
AÇÃO & 37,51 & 48,71 & 48,58 & 53,75 \\
HIDRAT. & & & & \\
$\begin{array}{l}\text { Qualidade } \\
\text { Global }\end{array}$ & $\mathbf{1 9 , 2 2}$ & $\mathbf{5 0 , 2 6}$ & $\mathbf{4 9 , 3 7}$ & $\mathbf{4 7 , 3 1}$ \\
\hline
\end{tabular}

Tabela 6 - Médias Sabonetes em barra

\begin{tabular}{ccccccccc}
\hline AMOSTRA & $\mathbf{1}$ & $\mathbf{2}$ & $\mathbf{3}$ & $\mathbf{4}$ & $\mathbf{5}$ & $\mathbf{6}$ & $\mathbf{7}$ & $\mathbf{8}$ \\
\hline APARÊNCIA & 18,31 & 25,68 & 43,81 & 68,18 & 10,56 & 52,31 & 31,58 & 37,87 \\
ODOR & 5,39 & 25,62 & 46,37 & 17,54 & 3,14 & 41,54 & 37,83 & 32,31 \\
ESPUMA & $-20,6$ & 23,83 & 25,64 & 7,14 & 69,29 & 43,77 & -1 & 15,27 \\
LIMPEZA & 47,14 & 48,58 & 19,72 & 31,58 & 51,54 & 36 & 49,33 & 46 \\
AÇÃO HID. & 9,7 & 21,16 & 1,56 & 6,83 & 43 & 58,54 & 35,26 & 49,35 \\
Qualidade & $\mathbf{1 1 , 9 9}$ & $\mathbf{2 8 , 9 7}$ & $\mathbf{2 7 , 4 2}$ & $\mathbf{2 6 , 2 5}$ & $\mathbf{3 5 , 5 0}$ & $\mathbf{4 6 , 4 3}$ & $\mathbf{3 0 , 6}$ & $\mathbf{3 6 , 1 6}$ \\
\hline
\end{tabular}

O efeito das variáveis nas formulações foi avaliado pelo software Statisca com base na tabela ANOVA e com gráficos de resíduos.

Análise dos efeitos das variáveis sobre os quesitos avaliados - Sabonete Líquido

Tabela 7 - Análise do efeito da aparência

\begin{tabular}{lllll}
\hline & SS & MS & F & p \\
\hline Intercepto & 123147.0 & 123147.0 & 42.04415 & 0.000000 \\
VolOl & 48570.1 & 48570.1 & 16.58252 & 0.000085 \\
VolEsp & 2282.5 & 2282.5 & 0.77926 & 0.379192 \\
VolOI*VolEsp & 12722.4 & 12722.4 & 4.34359 & 0.039344 \\
Erro & 339763.2 & 2929.0 & & \\
\hline
\end{tabular}

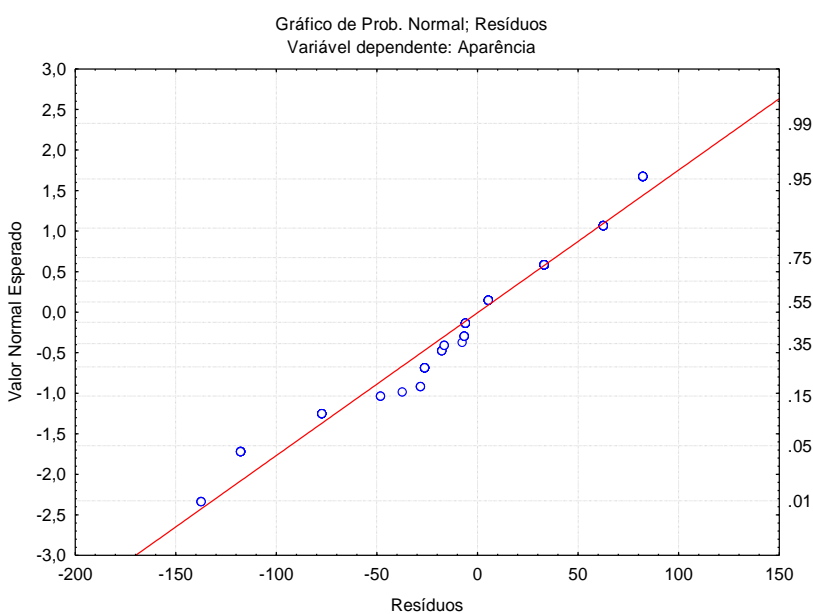

\section{Figura 5 - Gráfico de resíduos do efeito da} aparência.

Esta análise apresentada na Tabela 7 e Figura 5 foi realizada da mesma forma para os efeitos odor; limpeza; espuma e ação hidratante. 
Análise dos efeitos das variáveis sobre os quesitos avaliados - Sabonete em barra

Tabela 8 - Análise do efeito da aparência

\begin{tabular}{lllll}
\hline & SS & MS & F & p \\
\hline Intercepto & 311707.1 & 311707.1 & 121.3034 & 0.000000 \\
VolEsp & 2100.5 & 2100.5 & 0.8174 & 0.366867 \\
VolOl & 20861.5 & 20861.5 & 8.1184 & 0.004776 \\
mArg & 23877.0 & 23877.0 & 9.2919 & 0.002569 \\
VolEsp*VolOl & 14146.3 & 14146.3 & 5.5051 & 0.019803 \\
VolEsp*mArg & 994.9 & 994.9 & 0.3872 & 0.534406 \\
VolOI*mArg & 1277.7 & 1277.7 & 0.4972 & 0.481427 \\
VolEsp*VolOI*mArg & 10318.6 & 10318.6 & 4.0156 & 0.046245 \\
Erro & 596158.2 & 2569.6 & & \\
\hline
\end{tabular}

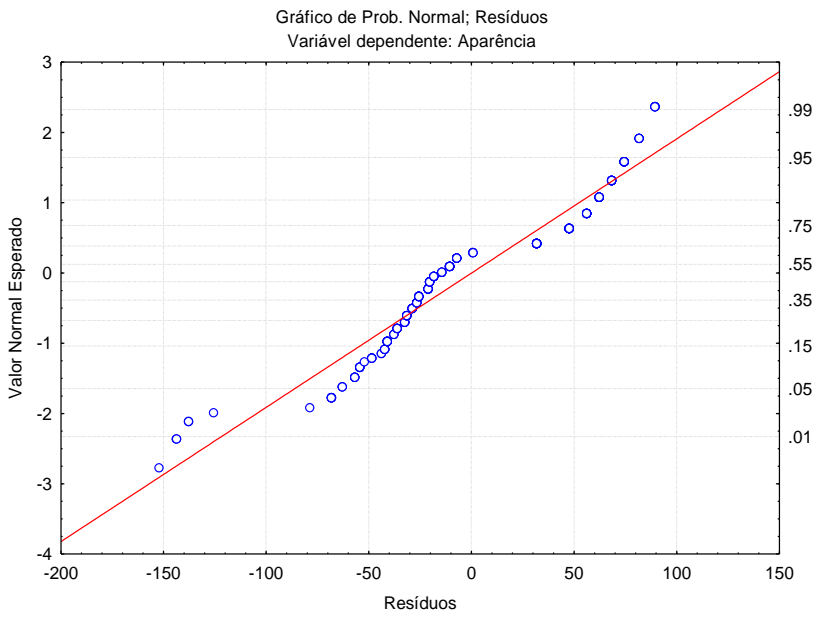

Figura 6 - Gráfico de resíduos do efeito da aparência.

Esta análise apresentada na Tabela 8 e Figura 6 foi realizada da mesma forma para os efeitos odor; limpeza; espuma e ação hidratante.

\section{CONCLUSÃO}

A partir do valor de qualidade global a amostra de maior aceitação para os sabonetes líquidos pode ser identificada, esta foi a de formulação $2(-1+1)$, que apresenta o índice inferior de volume de óleo e o superior de volume de anfótero. $\mathrm{Na}$ análise dos sabonetes em barra a amostra de maior aceitação foi a de formulação $6(-1+1-1)$, que apresenta o índice inferior de volume de óleo, índice superior de volume de espumógeno e índice inferior de massa de argila.

A partir do software estatístico pode-se avaliar os principais efeitos em cada quesito avaliado, assim como, por exemplo, para o sabonete líquido, na variável aparência foi visualizado que o volume de óleo e a interação entre o volume de óleo e de anfótero têm maior significância sobre estas variáveis. Estas análises foram feitas da mesma forma para os demais quesitos avaliados e também para a formulação de sabonetes em barra.

Feita todas essas análises, espera-se contribuir com a geração de conhecimento científico e tecnológico, utilizando esta metodologia no desenvolvimento de novos produtos de macadâmia produzidos no Espírito Santo.

\section{REFERÊNCIAS}

ABNT. Associação Brasileira de Normas Técnicas (1998), NBR 14141: escalas utilizadas em análise sensorial de alimentos e bebidas. Rio de Janeiro.

AGROSOFT BRASIL. (2009), Disponível em: <http://www.agrosoft.org.br/agropag/2142 68.htm>. Acesso em 3 de maio de 2011.

DIERBERGER, J.E.; MARINO NETTO, L.M. (1985), Noz macadâmia: uma nova opção para a fruticultura brasileira. São Paulo: Nobel,120 p.

IFT. Institute of Food Tchnologists (1981), "Sensory evaluation guide for testing food and beverage products". Food Tecnhology, v. 35 , n. 11 , p. 50-57, nov. Chicago.

KOTLER, P.; A., G. (1993), Princípios de

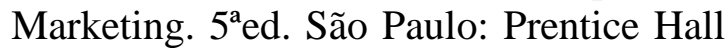
do Brasil.

LAND, D. G.; SHEPHERD, R. (1988), Scaling and ranking methods. In: Piggott, J. R. Sensory analysis of foods. New York: Elsevier Applied Science, p. 155170.

WERKEMA, M. C. C. (1996), Aguiar, S. Planejamento e análise de experimentos. Série Ferramentas da Qualidade. Belo Horizonte: FCO, 294 p.

\section{AGRADECIMENTOS}

Ao apoio financeiro recebido da UFES. Aos orientadores Leonardo Arrieche e Demerval Sartori pelo direcionamento. 